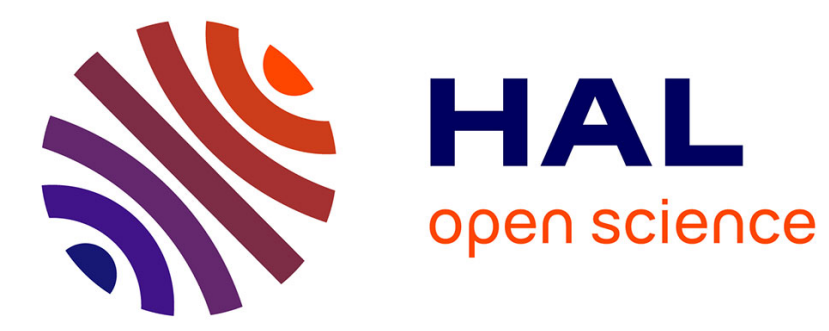

\title{
The influence of incoming turbulence on the dynamic modes of an NREL-5MW wind turbine wake
}

Giovanni de Cillis, Stefania Cherubini, Onofrio Semeraro, Stefano Leonardi, Pietro de Palma

\section{- To cite this version:}

Giovanni de Cillis, Stefania Cherubini, Onofrio Semeraro, Stefano Leonardi, Pietro de Palma. The influence of incoming turbulence on the dynamic modes of an NREL-5MW wind turbine wake. Renewable Energy, 2022, 183, pp.601 - 616. 10.1016/j.renene.2021.11.037 . hal-03498440

\author{
HAL Id: hal-03498440 \\ https://hal.science/hal-03498440
}

Submitted on 6 Jan 2022

HAL is a multi-disciplinary open access archive for the deposit and dissemination of scientific research documents, whether they are published or not. The documents may come from teaching and research institutions in France or abroad, or from public or private research centers.
L'archive ouverte pluridisciplinaire HAL, est destinée au dépôt et à la diffusion de documents scientifiques de niveau recherche, publiés ou non, émanant des établissements d'enseignement et de recherche français ou étrangers, des laboratoires publics ou privés. 


\title{
The influence of incoming turbulence on the dynamic modes of an NREL-5MW wind turbine wake
}

\author{
Giovanni De Cillis ${ }^{1}$, Stefania Cherubini ${ }^{1, *}$, Onofrio Semeraro ${ }^{3}$, Stefano Leonardi $^{2}$, Pietro De \\ Palma ${ }^{1}$
}

\begin{abstract}
Knowledge of the dynamics of wind turbine wakes and its dependence on the incoming boundary layer is fundamental to optimize and control the power production of wind farms. This work aims at investigating the effect of inflow turbulence on the wake of the NREL5MW wind turbine. Sparsity-Promoting Dynamic Mode Decomposition (SP-DMD) is performed on snapshots extracted from large-eddy simulations of the turbine wake, for detecting the most dynamically-relevant flow structures in the presence or absence of inflow turbulence. We demonstrate that inflow turbulence generated by a precursor simulation radically changes the most dynamically-relevant flow structures. For the laminar-inflow case the DMD modes selected by the SP algorithm have high wavenumbers and are spatially localized. When turbulence is added at the inflow, these high-frequency modes are superseded by low-frequency modes lying in the frequency range of the wake meandering and filling the whole domain, mostly corresponding to those dynamically relevant for the precursor simulation. These results show that, in the presence of inflow turbulence, coherent structures linked to endogenous mechanisms such as tip and root vortices loose their dynamical relevance in favour of those exogenously excited by turbulence, indicating that low-dimensional models of turbine wakes should take into account atmospheric turbulence.
\end{abstract}

Keywords: NREL 5MW wind turbine, DMD modes, uniform or turbulent inflow, turbulent precursor simulation

\section{Introduction}

Wind energy represents one of the fundamental renewable energy source to meet the imminent climate goals provided for 2050, with an expected electricity production of about $35 \%$ of the total demand [1]. To reach this goal, an increase of the actual wind energy production is needed, which can be achieved by increasing the number of installed wind turbines and/or the size of the rotor disks and, at the same time, by improving the efficiency of new turbines and wind farms. Such an acceleration in the deployment of wind power installations requires the enhancement of policy support initiatives, eradication of regulatory uncertainties, and further investments in new technologies [2]. Concerning the wind turbine innovation, the trend towards increasing rotor diameters and hub heights is leading to a single-unit power production of 5-6 MW in the case of onshore applications (from an average of 2.6 MW in 2018), using diameters of 160-170 m. In the case of offshore installations, a single wind turbine will achieve 15-20 MW in the next 10-20 years, with diameters of about $230 \mathrm{~m}$ and hub heights of $150 \mathrm{~m}$. Moreover, the optimization of the farm design, concerning, for instance, the turbine layout, and

*stefania.cherubini@poliba.it

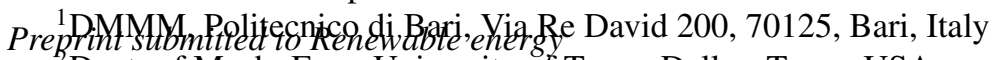

${ }^{2}$ Dept. of Mech. Eng., University of Texas, Dallas, Texas, USA

January 6, 2022

${ }^{3}$ LISN, Université de Paris-Saclay, CNRS, Orsay, France 
its control become crucial to guarantee high efficiency, flexibility and security of the power generation.

When clustered into farms, wind turbines are aerodynamically coupled to each-other in a very complex way through the action of their wakes [3,4]. A turbine in the wake of another may produce up to $40 \%$ less energy depending on the distance between them and on the turbulence intensity of the wind. The interaction becomes stronger when one attempts to increase the power density of the plant by reducing the distance among the turbines. Several innovative approaches can be exploited to optimize the farm efficiency and power density by controlling the axial induction $[5,6]$ or the wake direction $[7,6]$ of each turbine. In particular, strategies based on adjusting the rotor yaw angle have recently been proposed [7]. This technique consists of creating a yaw misalignment between the turbine rotor and the incoming wind, so that the wake is deflected laterally. As a consequence of the yaw misalignment, the efficiency of the upstream turbine decreases, but the power production of the downstream turbine increases due to the lateral deflection of the incoming wake. Another technique consists of tilting the rotor axis away from the mean wind direction in the vertical plane, so that the wake of the front turbine is redirected towards the ground, increasing the mean wind available to downstream turbines. A combined effect of optimal variation of yaw and tilt angles has been shown to guarantee a power increase of about $15 \%$ for two-row wind-aligned array of turbines [6].

Moreover, one has to consider that the hub heights of innovative wind turbines are higher then $100 \mathrm{~m}$, so that the rotors are immersed in the atmospheric boundary layer (ABL) and the incoming flow is characterized by a streamwise velocity gradient along the vertical direction. Such a gradient influences the blade loading, increasing the load on the blades pointing upwards in the ABL [8]. Therefore, any attempt of optimizing the performance of a wind farm, taking into account the interactions among the turbines, should include the effect of the shear of the $\mathrm{ABL}$ and its modifications. In fact, the wake produced by a wind turbine is characterized by different flow structures which can be originated by endogenous or exogenous mechanisms. The flow structures triggered by endogenous mechanisms are those produced by an isolated turbine subject to a laminar inlet velocity, such as the tip- and root-vortex helices or the von Kármán street developing behind the tower, as well as other structures arising downstream. However, the wake of a wind turbine could be significantly influenced by flow structures embedded in the ABL. In fact, coherent structures are detected in wind turbine wakes, which are not directly generated by the turbine but are triggered by exogenous phenomena. These coherent structures are different from those due to endogenous mechanisms and from those embedded in the ABL, since they are generated by the interaction between the ABL and the turbine. The nature of the incoming flow has a relevant effect on the operational state of the turbine and on the dynamics of its wake, including the main features of the turbulence generated by the turbine and the recovery of the wake. In particular, numerical [9] and experimental data $[10,11]$ indicate that single-turbine wakes recover faster with higher turbulence intensity levels in the incoming flows. Moreover, in the case of uniform inflow, the interaction between the incoming wind and the turbine generates an axisymmetric shear layer with axisymmetric turbulence statistics [9]. Instead, the typical non-uniform ABL velocity profile (logarithmic for neutral conditions) and its turbulence distribution induce a higher level of turbulent kinetic energy production in the upper part of the wake, due to the shear [12]. However, the influence of atmospheric turbulence on the development of coherent structures in the wake, originated by endogenous or exogenous mechanisms, has not been investigated in detail.

The present work aims at filling this gap by assessing or by quantifying the effect of a 
turbulent incoming flow on the coherent structures characterising the wake of the NREL-5MW reference wind turbine using a Sparsity-Promoting Dynamic Mode Decomposition (SP-DMD) for the detection of dynamically-relevant flow structures. The flow through the NREL-5MW wind turbine has been chosen as a realistic reference test case for a MW-scale wind turbine, since the details of the turbine's geometry and a complete set of flow data are extensively documented in literature and used in previous studies [13]. The DMD method was proposed by Schmid [14]. The mathematical meaning of DMD modes is discussed in Ref. [15], where the DMD modes are interpreted as a data-driven approximation of the Koopman modes, based on a sequence of flow-field snapshots. The DMD method consists of finding the eigenvalues and the eigenvectors of a linear operator approximating the non-linear dynamics embedded in the data sequence and it has been recently exploited for the formulation of reduced order models (ROMs) of wind-turbine flows [16, 17, 18]. DMD is applied to time-resolved data, such as a sequence of snapshots obtained from numerical or experimental analyses, to determine the dominant mechanisms characterizing the dynamics of the flow, namely, the DMD modes. Such modes are non-orthogonal and are characterized by a single time frequency, so that they can be sorted by their spectral contribution and spatial growth rate. This can be particularly interesting for the design of a wind farm layout, since one can analyse which modes conserve a relevant energy contribution downstream of a wind turbine and which frequencies are involved in the dynamic load acting on downstream turbines. The interested reader can find further details on the numerous DMD variants in Ref. [19].

DMD has been recently applied to the flow over a wind turbine. In particular, Sarmast et al. [20] employed the DMD approach to study numerically a single wind turbine wake, focusing on the tip-vortex instability onset, using the actuator line method. In Ref.s $[16,17,18]$, reducedorder models of wind turbine wakes were developed, using the DMD method based on Large Eddy Simultaion (LES) flow data and considering different operational regimes. Premaratne $\& \mathrm{Hu}$ [21] provided an experimental study of the flow behind a horizontal-axis wind turbine subject to a typical atmospheric boundary layer wind under neutral stability conditions. A highresolution digital particle image velocimetry was employed to obtain the snapshot sequence, which was analyzed by the DMD method. Le Clainche et al. [18] employed the DMD method based on Lidar measurements to build a reduced-order model of the wind velocity upstream of a horizontal-axis wind turbine. Kleine et al. [22] provided a numerical study of the tip vortex instability in two in-line turbines using the actuator-line method and the DMD approach. Sun et al. [23] recently employed an LES to compute the unsteady flow over a two-blade wind turbine model. They analyzed the wake flow using the DMD method in the absolute and in the relative (with respect to the rotor) reference frames.

In general, only a limited number of DMD modes are relevant for the interaction among turbines, allowing one to simplify the interaction model for optimization purposes [16]. DMD modes are usually ranked according to their amplitude corresponding to the first snapshot of the data-sequence. Using such a criterion for sorting the modes and selecting a limited number of most significant modes can fail in capturing the relevant dynamics of the phenomena under consideration, leading to low-accurate predictive capability of the generated low-dimensional models. For this reason, several variants of the standard DMD algorithm, aiming at extracting a limited subset of relevant flow features that optimally approximate the original data sequence, have been developed, such as the Sparsity-Promoting-DMD [24]. In the present work we use such a version of the algorithm, for the first time in wind turbine wake characterization, for ranking the most relevant DMD modes. 
The aim of this study is to investigate the effect of incoming turbulence on the most relevant coherent structures embedded in the turbulent wake flow developing downstream of the NREL-5MW wind turbine, using the SP-DMD approach. The proposed analysis is particularly relevant to optimise the energy production capacity of wind farms, on which the dynamics of the turbine wakes has a critical effect. The snapshot data-base is obtained through LES, performed in the absence and in the presence of inlet turbulence The actuator line method is used for simulating the rotor, whereas, tower and nacelle are modeled with the immersed boundary method $[25,26]$. The wind-turbine flow is simulated firstly imposing a laminar uniform inlet velocity profile and then considering a turbulent incoming flow generated by a precursor simulation. The incoming turbulent wind profile and its statistics have been generated by a preliminary LES considering a staggered-cube-array roughness distribution at wall simulating the landscape canopies [27]. Sparsity-promoting DMD is performed on the turbine wake with both uniform and turbulent inlet conditions, and on the precursor simulation itself, for comparison. It is found that the presence of inlet turbulence strongly affects the spatial structure and temporal frequency of the selected DMD modes.

The paper is structured as follows. In Sect. 2, the LES and DMD framework is described. The flow settings for the precursor and turbine wake simulations are presented in Sect. 3. In Sect. 4, the results of DMD of the flow fields obtained by LES are presented and discussed. Finally, in Sect. 5, the main results are summarized and conclusions drawn.

\section{Methodologies}

\subsection{Large Eddy Simulation}

Large Eddy Simulation (LES) of the wake behind the NREL-5MW wind turbine is carried out by solving the following governing equations for the filtered non-dimensional velocity components, $u_{i}$, where $i \in\{1,2,3\}$ indicate the streamwise, $x$, wall-normal, $y$, and spanwise, $z$, directions and the pressure $p$ :

$$
\begin{gathered}
\frac{\partial u_{i}}{\partial t}+\frac{\partial u_{i} u_{j}}{\partial x_{j}}=-\frac{\partial p}{\partial x_{i}}+\frac{1}{R e} \frac{\partial^{2} u_{i}}{\partial x_{j} \partial x_{j}}-\frac{\partial \tau_{i j}}{\partial x_{j}}+F_{i} \\
\frac{\partial u_{i}}{\partial x_{i}}=0
\end{gathered}
$$

The Reynolds number is defined as $R e=\frac{U_{\infty} D}{\nu}$, where $U_{\infty}$ is the inlet velocity at the hub height, $D$ is the rotor diameter and $\nu$ is the kinematic viscosity of the fluid, which have been used as reference variables for non-dimensionalisation. Concerning the residual stress tensor, $\tau_{i j}$, while its isotropic part is included in the modified filtered pressure as $p^{*}=p+\frac{1}{3} \tau_{i i}$, its anisotropic part has to be modelled. Here we use the Smagorinsky model, based on the linear eddy viscosity hypothesis that relates the residual stress to the filtered rate of strain, $S_{i j}$, according to the following equation,

$$
\tau_{i j}^{r}=-2 \nu_{r} S_{i j}
$$

where the proportionality constant $\nu_{r}$ is the eddy viscosity of residual motions. The latter is, in turn, modelled using the mixing length hypothesis as:

$$
\nu_{r}=l_{S}^{2} \mathcal{S}=\left(C_{S} \Delta\right)^{2} \mathcal{S}
$$


where $\delta$ is the characteristic filtered rate of strain, defined as $\mathcal{S} \equiv\left(2 S_{i j} S_{i j}\right)$, and $l_{S}$ is the Smagorinsky lenght-scale, which is defined as the product between the Smagorinsky constant $C_{S}$ and the filter width $\Delta$. The Smagorinsky constant is set to 0.17 , whereas the filter width is defined as the cube root of the local cell volume.

The governing equations (1) are solved using a second-order-accurate centered finite difference scheme on a staggered Cartesian grid and a hybrid low-storage third-order-accurate Runge-Kutta scheme for time integration [28]. Note that the last term in equation (1), $F_{i}$, represents the force per unit volume given by the actuator line method [29], which models the forces exerted by the turbine blades on the fluid by means of the lift and drag coefficients $C_{L}, C_{D}$. Approximating the rotor blades as rotating rigid lines divided into discrete segments, each one associated with a relative inflow velocity, $u_{r e l}$, and an angle of attack, $\alpha$, one can estimate the lift and drag forces (per unit length) as:

$$
\begin{aligned}
F_{L} & =\frac{1}{2} \rho u_{r e l}^{2} C_{L}(\alpha) c F, \\
F_{D} & =\frac{1}{2} \rho u_{r e l}^{2} C_{D}(\alpha) c F,
\end{aligned}
$$

where $\rho$ is the fluid density, $c$ is the chord, $F$ is the modified Prandtl correction factor accounting for the aerodynamic effect of the tip and root vortices [25]. These forces are then spread on areas perpendicular to each actuator line using a Gaussian distribution kernel. The tower and nacelle are modeled by the immersed boundary method, using a a discrete forcing approach $[30,31]$. The overall method has been extensively validated in the case of wind turbines [25, 26]. The code has also been coupled with WRF to simulate a real wind farm in north Texas. Numerical results agreed very well with SCADA data [32].

\subsection{Sparsity Promoting Dynamic Mode Decomposition}

The Dynamic Mode Decomposition (DMD) [15, 14], is a data-driven technique capable of extracting dynamically relevant flow structures by assuming a linear mapping between successive snapshots. The variant employed in the present study is called Sparsity Promoting Dynamic Mode Decomposition (SP-DMD) and was proposed by Jovanović et al. [24]. The main idea is to carry out a constrained error minimization for optimally reconstructing the data-set using selecting only a limited number of DMD modes. The first step is to collect, from numerical simulations or experimental findings, snapshots of the flow field, $\boldsymbol{q}^{i} \in \mathbb{C}^{N}$, at a constant sampling frequency. Each snapshot has dimension $N=O \times S, O$ being the number of observables, $S$ representing the number of measurements points. Let us consider $M$ snapshots and assume a linear time-invariant mapping $\boldsymbol{A}$ that connects a successive pair of these snapshots as:

$$
\boldsymbol{q}^{i+1}=\boldsymbol{A q}^{i}, \quad i=\{0, \ldots, M-1\}
$$

Introducing the matrices

$$
\begin{aligned}
& \mathbf{Q}^{0}=\left[\boldsymbol{q}^{0} \boldsymbol{q}^{1} \ldots \boldsymbol{q}^{M-1}\right], \\
& \mathrm{Q}^{1}=\left[\boldsymbol{q}^{1} \boldsymbol{q}^{2} \ldots \boldsymbol{q}^{M}\right],
\end{aligned}
$$

and using equation (7) one has:

$$
\mathrm{Q}^{1}=A \mathrm{Q}^{0}
$$


For reducing the degrees of freedom of the problem, as suggested by Schmid [14], the operator $\boldsymbol{A}$ can be projected onto the $r$-dimensional POD basis $\mathbf{U}$ of the snapshots matrix $\mathbf{Q}^{0}$, calculated using a Singular Value Decomposition (SVD) or the snapshot method [33],

$$
\mathbf{Q}^{0}=\mathbf{U S V}^{*} \text {. }
$$

Then, the matrix $\boldsymbol{A}$ is approximated by

$$
\boldsymbol{A} \approx \mathbf{U} \boldsymbol{F} \mathbf{U}^{*}
$$

where the matrix $\boldsymbol{F}$ is obtained by minimizing the Frobenius norm of the difference between $\mathrm{Q}^{1}$ and $A \mathrm{Q}^{0}$, as:

$$
\min _{\boldsymbol{F}}\left\|\mathbf{Q}^{1}-\mathbf{U} \boldsymbol{F} \mathbf{S V}^{*}\right\|_{F}^{2}
$$

leading to the following optimal solution:

$$
\boldsymbol{F}=\mathbf{U}^{*} \mathbf{Q}^{1} \mathbf{V S}^{-1}
$$

Dynamic modes are then extracted by computing the eigendecomposition of the matrix $\boldsymbol{F}$ :

$$
\boldsymbol{F}=\underbrace{\left[\begin{array}{lll}
\boldsymbol{y}_{1} & \ldots & \boldsymbol{y}_{r}
\end{array}\right]}_{\boldsymbol{Y}} \underbrace{\left[\begin{array}{ccc}
\mu_{1} & & \\
& \ddots & \\
& & \mu_{r}
\end{array}\right]}_{\boldsymbol{D}_{\mu}} \underbrace{\left[\begin{array}{c}
\boldsymbol{z}_{1}^{*} \\
\vdots \\
\boldsymbol{z}_{r}^{*}
\end{array}\right]}_{\boldsymbol{Z}^{*}},
$$

$\boldsymbol{y}_{i}$ and $\boldsymbol{z}_{i}^{*}$ being the right and left eigenvectors of $\boldsymbol{F}$, normalized as $\boldsymbol{y}_{i}^{*} \boldsymbol{y}_{i}=1$ and $\boldsymbol{z}_{i}^{*} \boldsymbol{y}_{j}=\delta_{i j}$. Finally, the dynamics in the low-dimensional POD subspace, $\boldsymbol{x}^{i+1}=\boldsymbol{F} \boldsymbol{x}^{i}$ can be expressed as follows:

$$
\boldsymbol{x}^{n}=\boldsymbol{Y} \boldsymbol{D}_{\mu}^{n} \boldsymbol{Z}^{*} \boldsymbol{x}^{0}=\sum_{i}^{r} \boldsymbol{y}_{i} \mu_{i}^{n} \boldsymbol{z}_{i}^{*} \boldsymbol{x}^{0}=\sum_{i}^{r} \boldsymbol{y}_{i} \mu_{i}^{n} \alpha_{i},
$$

where $\alpha_{i}=\boldsymbol{z}_{i}^{*} \boldsymbol{x}^{0}$ represents the component of the initial condition in the POD subspace, $\boldsymbol{x}^{0}$, in the $\boldsymbol{z}_{i}^{*}$ direction. Thus, the snapshots can be expanded as a linear combination of the DMD modes $\phi_{i}=\mathbf{U} \boldsymbol{y}_{i}$ as:

$$
\boldsymbol{q}^{n} \approx \mathbf{U} \boldsymbol{x}^{n}=\sum_{i}^{r} \mathbf{U} \boldsymbol{y}_{i} \mu_{i}^{n} \alpha_{i}=\sum_{i}^{r} \boldsymbol{\phi}_{i} \mu_{i}^{n} \alpha_{i},
$$

where $\alpha_{i}$ is the amplitude of the corresponding DMD mode. Equation (17) can be written in matrix form:

$$
\underbrace{\left[\begin{array}{llll}
\boldsymbol{q}^{0} & \boldsymbol{q}^{1} & \ldots & \boldsymbol{q}^{M-1}
\end{array}\right]}_{\mathbf{Q}^{0}} \approx \underbrace{\left[\begin{array}{llll}
\boldsymbol{\phi}_{1} & \boldsymbol{\phi}_{2} & \ldots & \boldsymbol{\phi}_{r}
\end{array}\right]}_{\boldsymbol{\boldsymbol { D }}} \underbrace{\left[\begin{array}{llll}
\alpha_{1} & & & \\
& \alpha_{2} & & \\
& & \ddots & \\
& & & \alpha_{r}
\end{array}\right]} \underbrace{\left[\begin{array}{cccc}
1 & \mu_{1} & \ldots & \mu_{1}^{M-1} \\
1 & \mu_{2} & \ldots & \mu_{2}^{M-1} \\
\vdots & \vdots & \ddots & \vdots \\
1 & \mu_{r} & \ldots & \mu_{r-1}^{M}
\end{array}\right]}_{\boldsymbol{V}_{\text {and }}},
$$

where $\boldsymbol{V}_{\text {and }}$ is the Vandermonde matrix and $\boldsymbol{\Phi}:=\mathbf{U} \boldsymbol{Y}$ is the matrix composed of the DMD modes $\phi_{i}$. The associated amplitudes $\alpha=\left[\alpha_{1} \ldots \alpha_{r}\right]^{T}$ are then computed by minimizing the 
Frobenius norm of the difference between the left and the right size of equation (18), which can be performed in the low-dimensional POD subspace by using the eigendecomposition in equation (15), as:

$$
\min _{\alpha} J(\alpha)=\left\|\mathbf{S V}^{*}-\boldsymbol{Y} \boldsymbol{D}_{\alpha} \boldsymbol{V}_{\text {and }}\right\|_{F}^{2} .
$$

Superposing the computed DMD modes, weighted by their amplitudes and oscillating at the obtained frequency and growth rate, allows one to optimally approximate the data sequence. However, when aiming at finding a low dimensional representation of the snapshots' sequence, the objective function $J(\alpha)$ in (19) should be augmented with an additional term that penalizes the number of non-zero elements in the amplitudes' vector $\alpha$, as:

$$
\min _{\alpha} J(\alpha)+\gamma \sum_{i=1}^{r}\left|\alpha_{i}\right|,
$$

where $\gamma$ is a parameter that influences the sparsity level, higher values of the parameter promoting sparser solutions. The resulting sparsity-promoting DMD problem in equation (20) is a convex optimization problem, solved using the Alternating Direction Method of Multipliers [24].

\section{Simulation layout}

\subsection{Precursor simulation}

In order to mimic a turbulent ABL impinging on the turbine, the precursor simulation is performed at $R_{\text {bulk }}=U_{b u l k} D / \nu=10^{8}$, which is the same value used for the turbine wake flow simulation with uniform laminar inlet. The computational domain has dimensions $6 \times 5 \times 3$ diameter units in the streamwise, vertical and spanwise directions, respectively. No-slip boundary conditions are imposed at the bottom wall, whereas, at the top wall, vertical velocity and shear are set to zero. Periodicity is imposed in streamwise and spanwise directions. A staggered array of cubes with height $h_{c}=0.1$ diameters is placed at the bottom wall of a turbulent channel [27] to trigger turbulence. Figure 1 provides a detailed description of the cubes arrangement, whereas figure 2(a) provides a cross section of the time-averaged streamwise velocity contours, showing the influence of the cubes at the wall and the presence of large-scale flow structures. Note that the bottom wall is placed at $y=-0.714$ diameters, in order to have the origin of the vertical direction corresponding to the hub height in the turbine wake simulation. The timeand space-averaged (in a cross section) streamwise velocity profile provided in the left panel of figure 2 (b) appears well fitted with a logarithmic law in the inner region, except for $y<-0.65$, where the roughness elements are present. Whereas, the profile in the outer region is well described by a power law having shear coefficient $\alpha=0.27$. The turbulence intensity $(I)$ profile is provided in the right panel of Figure 2 (b) and shows a turbulence intensity of approximately $10 \%$ at the hub height. The corresponding time-varying turbulent velocity obtained by the precursor simulation is imposed at the inlet of the turbine flow computational domain, as specified in the following.

\subsection{Turbine flow LES}

We consider the NREL-5MW wind turbine with a tip-speed ratio $\lambda=7$ and Reynolds number $R e=10^{8}$. The computational domain has dimensions $12.5 \times 5 \times 3$ diameters in $x, y, z$, respectively, as shown in figure 3 . The turbine is centered in the transverse direction, 

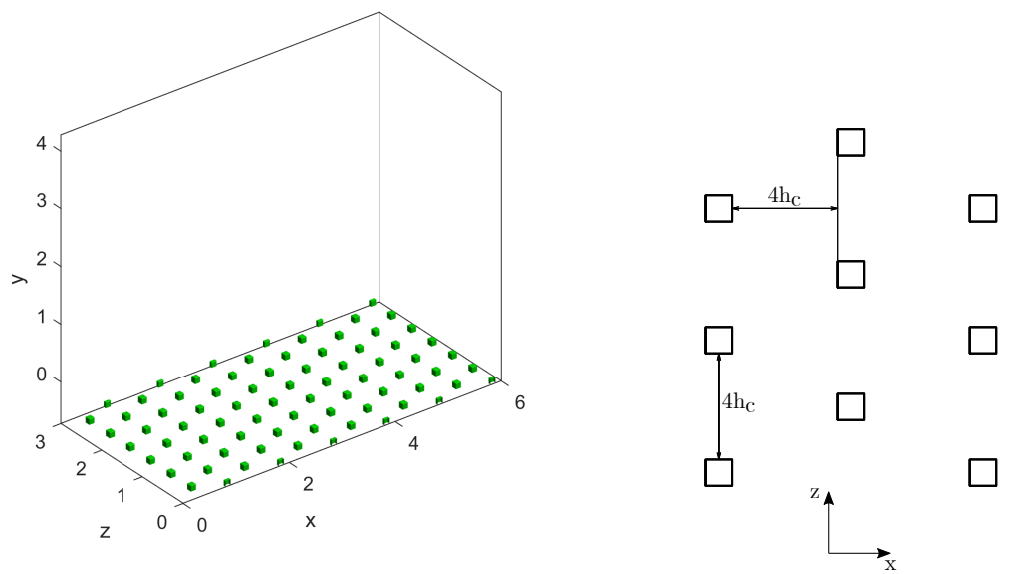

Figure 1: Layout of the precursor simulation reporting the arrangement of the cubes mounted at the wall.

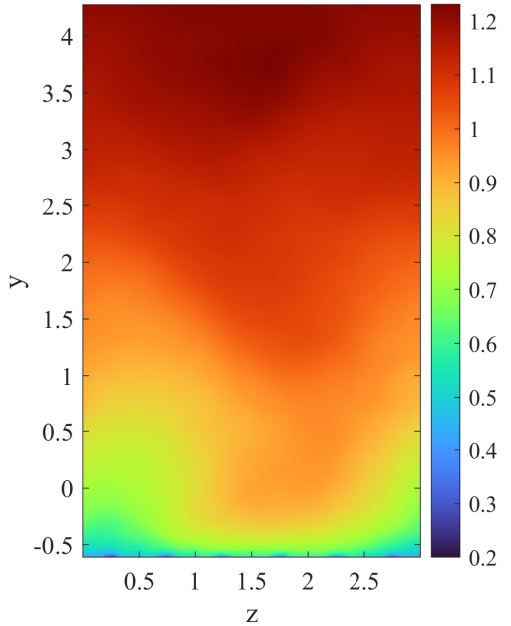

(a)

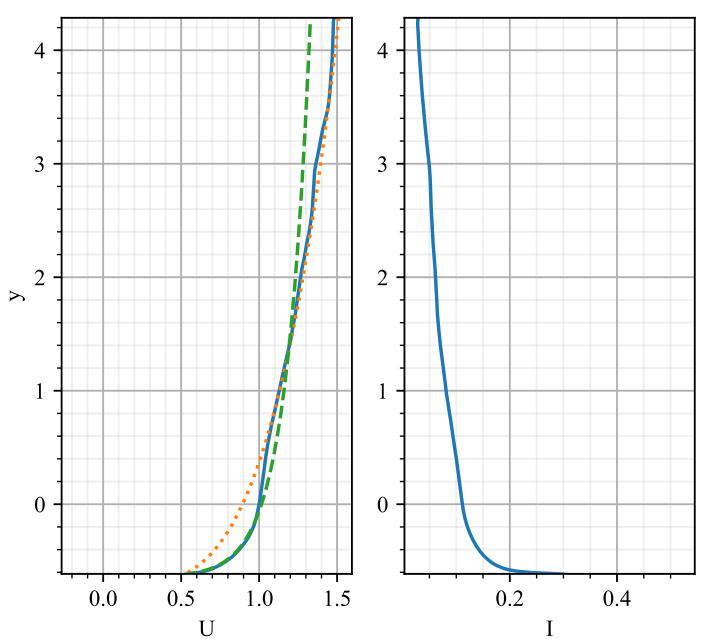

(b)

Figure 2: (a) Time-averaged streamwise velocity contours in a cross section of the precursor simulation computational domain. (b) Time- and space-averaged axial velocity and turbulent intensity profiles of the precursor (blue solid lines). The green dashed line is the log-law and the orange dotted line the power law of the wind profile. Note that in both figures, the region below the cubes' height is not shown. 


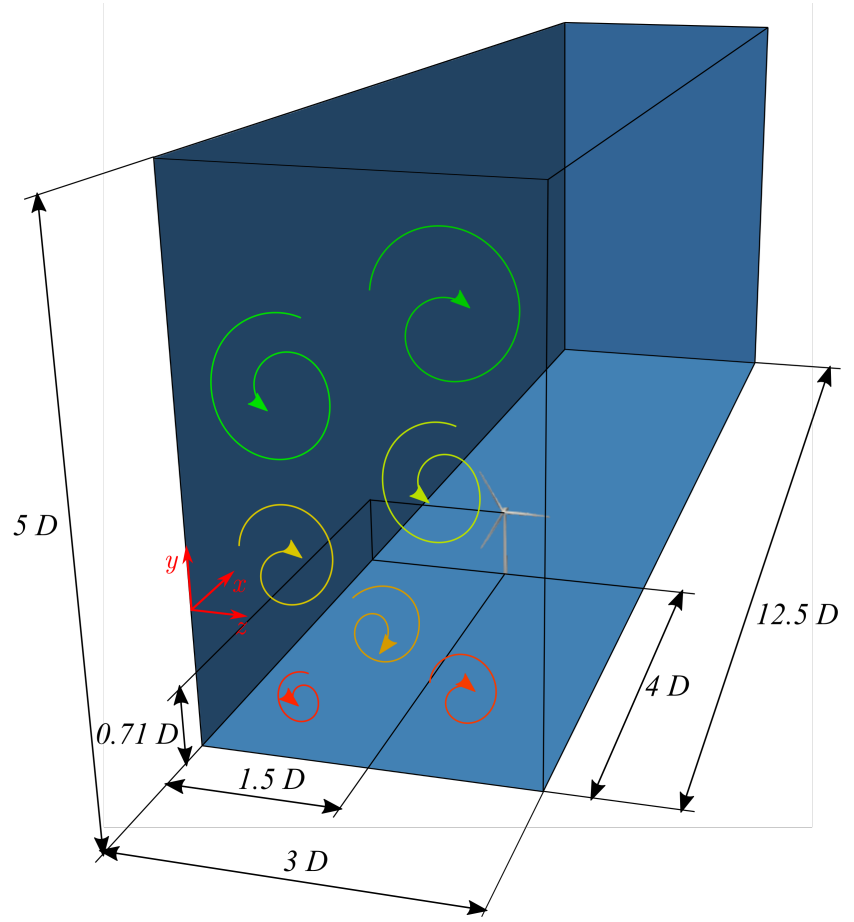

Figure 3: Computational domain and setting of the turbine wake LES. The coloured vortices represent the turbulent inflow.

and is located at 4 diameters from the inlet, where a uniform velocity $U_{\infty}$ or the previously obtained turbulent profile is imposed. Note that in the latter case, due to turbulence decay, the turbulence intensity that impinges on the turbine is lower than $10 \%$ at the hub height. The computational domain is discretised by grid consisting of $2048 \times 512 \times 512$ grid-points in the $x, y, z$ directions, respectively. The grid is uniform along the streamwise and transverse directions, and it is stretched in the vertical direction, with finer grid spacing in the turbine wake region.

\section{DMD results}

\subsection{Turbine wake with uniform inflow}

The DMD analysis of the wake of the NREL-5MW wind turbine with uniform incoming flow has been discussed in detail in Ref. [34]. We provide here the main results, which are relevant for the comparison with the case of a turbulent incoming flow. Snapshots of the velocity field are extracted in a reduced three-dimensional subdomain of extent $[0-8.4] \times[-0.7-$ $0.63] \times[0.8-2.2]$ in the $x, y$ and $z$ direction, respectively, every $\Delta t$ corresponding to a $10^{\circ}$ rotation of rotor. The dataset is made by 3052 snapshots, whose ensemble average is subtracted from each snapshot before applying the DMD procedure. As a consequence, all the eigenvalues lie on the unitary circle, as shown in the left panel of figure 4, providing the eigenvalues, $\mu$, of the linear operator $\boldsymbol{F}$ (see equation (15)). The frequency and growth rate of the DMD modes are shown in the right panel of figure 4 . The modes selected by the sparsity-promoting algorithm for $\gamma=40000$ are marked with a black circle. For this high value of $\gamma$, five pairs of DMD modes are selected, which are shown in figure 5. The second frame shows the mode having $\mathfrak{R}(\omega)=42$, which is clearly related to the tip vortices and oscillates at an angular frequency equal to three times that of the turbine (due to the number of blades). The remaining 


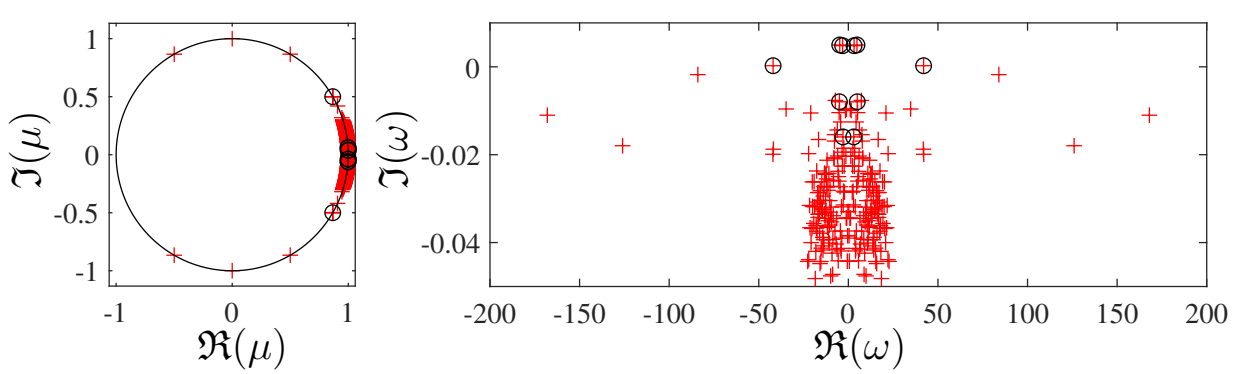

Figure 4: Eigenvalues resulting from the standard DMD algorithm (red crosses) and the sparsity promoting algorithm (black circles) for the case with laminar uniform inflow. The right panel shows the logarithmic mapping of the eigenvalues, $\omega=-\frac{\log (\mu)}{i \Delta t}$, where $\Delta t$ is the time interval between two consecutive snapshots and $i$ is the imaginary unit.

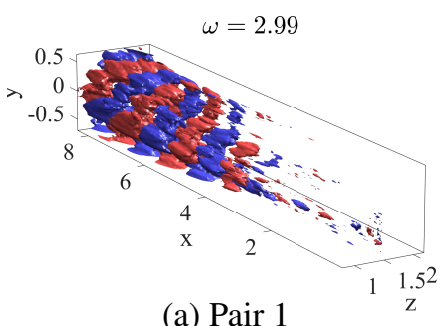

(a) Pair 1

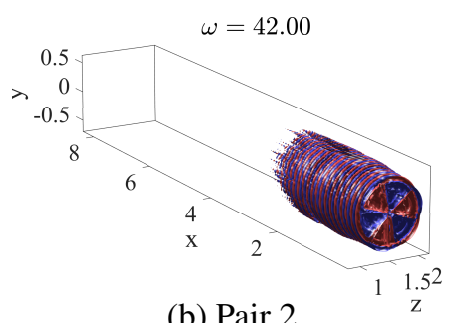

(b) Pair 2

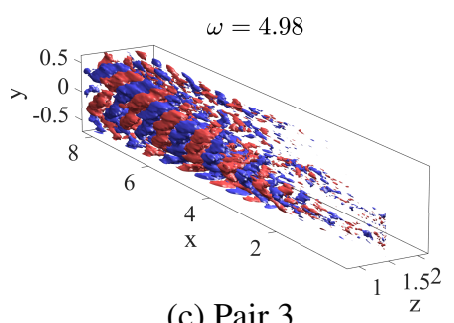

(c) Pair 3

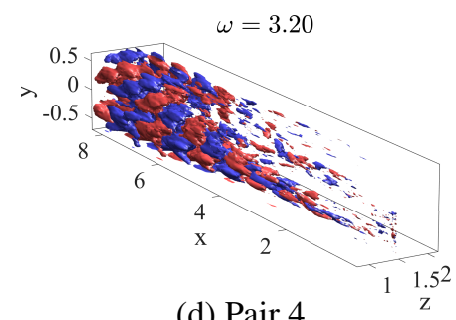

(d) Pair 4

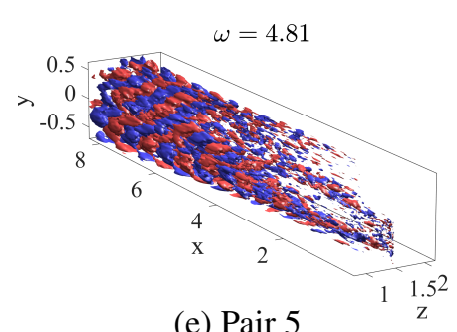

(e) Pair 5

Figure 5: Streamwise velocity iso-surfaces (red for positive, blue for negative values) of the real part of the 5 dynamic modes' pairs selected by the sparsity-promoting algorithm for $\gamma=40000$ and for the laminar uniform inflow, ordered according to their amplitude $|\alpha|$.

selected modes, having lower frequencies in the range $\mathfrak{R}(\omega) \in[2.99-4.97]$, are associated to spatial structures with moderate length scale, localized in the far wake region (see figure $5(\mathrm{a}, \mathrm{c}-\mathrm{e})$ ). For lower values of $\gamma$, as shown by the amplitude spectrum in figure 6 , many other low-frequency modes are found. These modes present a spatial structure (not shown) similar to the moderate-scale modes previously shown, but associated to slightly higher/lower temporal wavenumbers. High-frequency modes are present as well, mostly appearing as harmonics of the previously shown DMD mode associated to the tip vortices, provided in figure 5 (b). Some moderate-frequency modes $(\mathfrak{R}(\omega) \in[10-20])$ are present as well, mostly localized in the tower's and nacelle's wake and characterized by a fine spatial structures (not shown). A thorough discussion of these modes, which are used here as reference for the next considered cases, can be found in Ref. [34].

\subsection{Precursor simulation}

The DMD analysis has been applied to the precursor LES results by extracting the snapshots every $\Delta t=0.033$ in a subdomain of size $[0-6.0] \times[-0.7-0.63] \times[0.8-2.2]$ in the $x, y$ and $z$ direction, respectively. The complete data-set is composed of 2990 snapshots. The convergence 


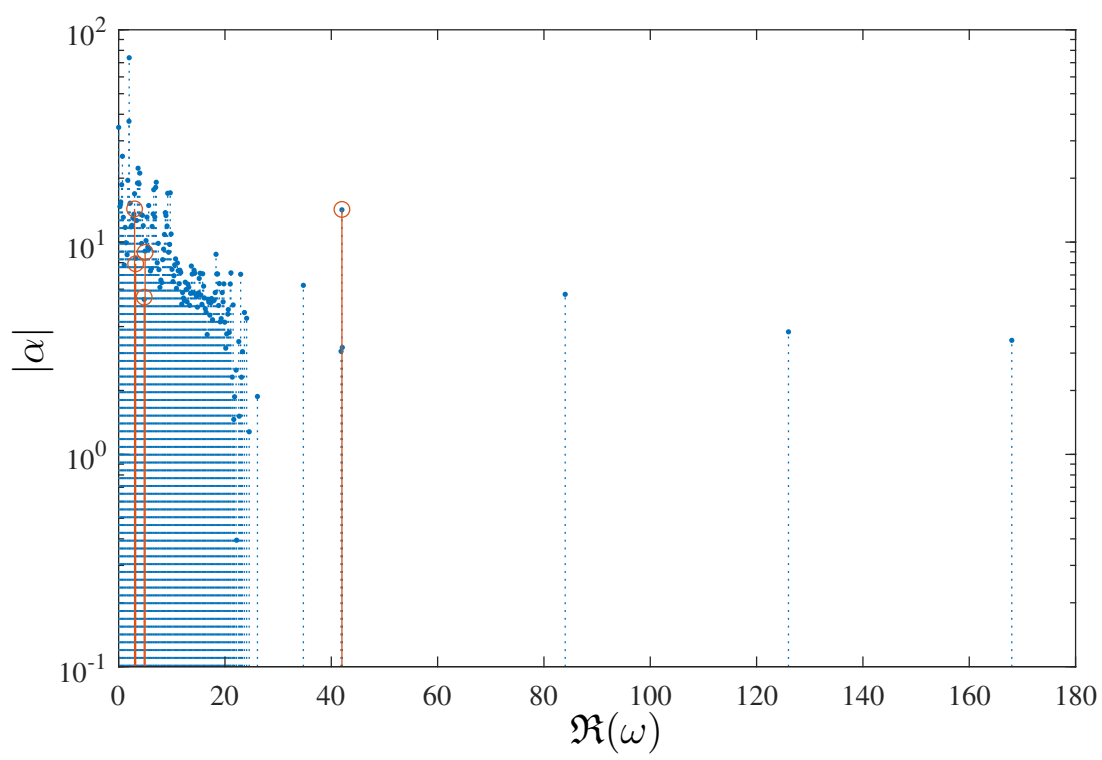

Figure 6: Dependence of the absolute value of the DMD amplitudes $\alpha_{i}$ on the angular frequency $\Re(\omega)$, for the case with laminar inflow. The results are obtained using the standard (blue stems) and the sparsity-promoting algorithm with $\gamma=40000$ (orange stems). Due to the symmetry of amplitudes' distribution, only positive frequencies are shown.

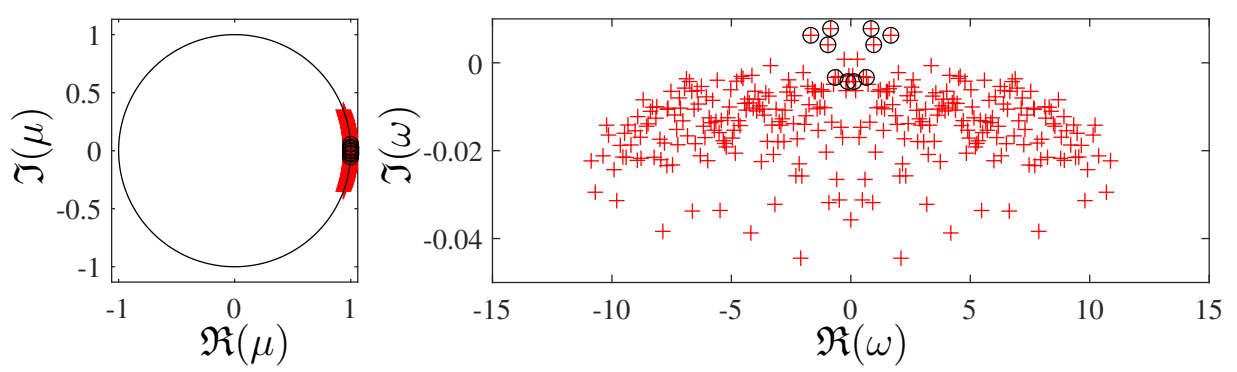

Figure 7: Eigenvalues resulting from the standard DMD algorithm (red crosses) and the sparsity promoting algorithm (black circles) for the precursor simulation. The right panel shows the logarithmic mapping of the eigenvalues, $\omega=-\frac{\log (\mu)}{i \Delta t}$, where $\Delta t$ is the time interval between two consecutive snapshots and $i$ is the imaginary unit.

study, reported in the Appendix, indicates a good stabilization of the frequency values already for a POD subspace of dimension $r \geq 251$, whereas the growth rates are well approximated for $r>300$. For this reason, in the following, we will use a POD basis of dimension $r=301$. In the left panel of Figure 7, the eigenvalues $\mu$ of the linear operator $\boldsymbol{F}$ are provided, along with the unit circle. The modes selected for $\gamma=65000$ are marked with a black circle. Looking at the right frame of figure 7, providing the temporal wavenumber $\mathfrak{R}(\omega)$ of the modes, one can note that the DMD modes are all characterized by rather low frequencies $(|\Re(\omega)|<10)$ compared to the previous case, which reaches wavenumbers of order $10^{2}$. The amplitude of these low-frequency modes is provided in figure 8, showing that the highest amplitudes are concentrated at the lowest frequencies. The red circles indicate the five modes selected by the sparsity-promoting algorithm for $\gamma=65000$. The spatial structures of these five selected pairs are shown in figure 9 .

The lowest-frequency mode with $\mathfrak{R}(\omega)=0.13$, shown in figure 9 (a), is characterized by 


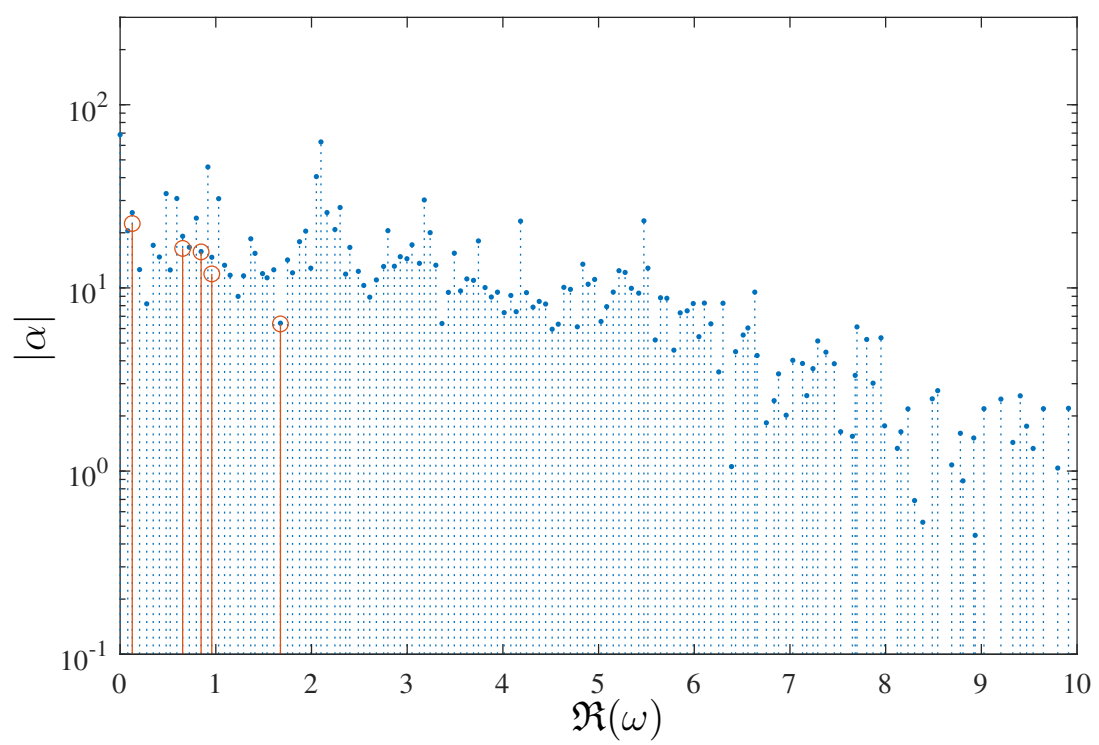

Figure 8: Dependence of the absolute value of the DMD amplitudes $\alpha_{i}$ on the angular frequency $\mathfrak{R}(\omega)$, for the precursor simulation. The results are obtained using the standard (blue stems) and the sparsity-promoting algorithm (orange stems). Due to the symmetry of amplitudes' distribution, only positive frequencies are shown.

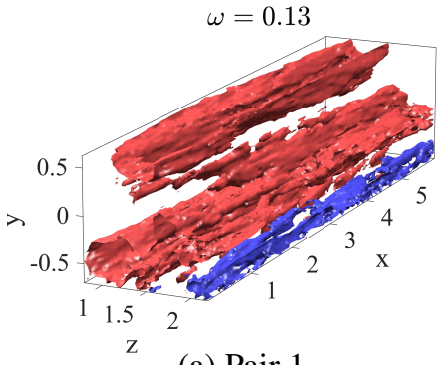

(a) Pair 1

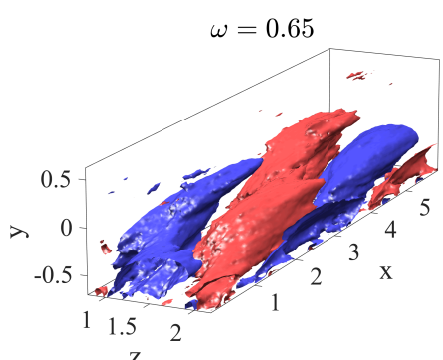

(b) Pair 2

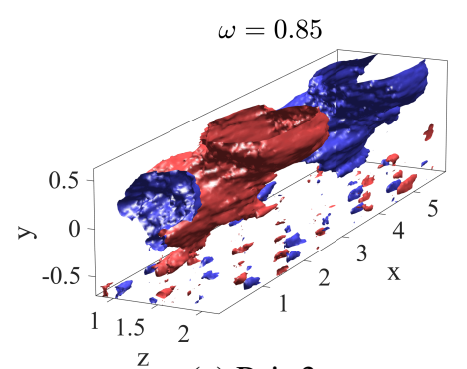

(c) Pair 3

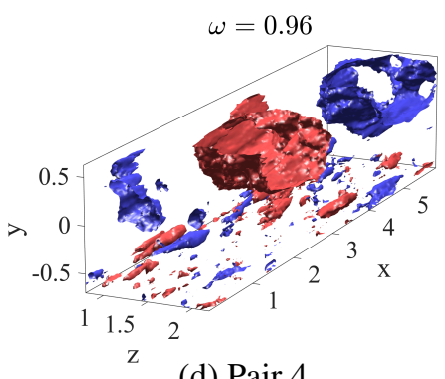

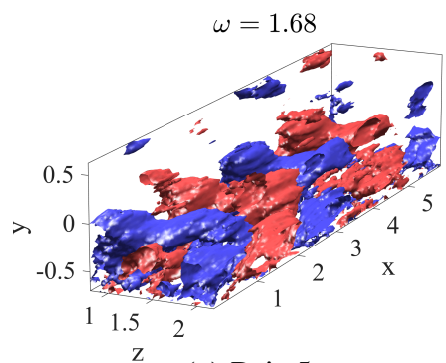

(e) Pair 5

Figure 9: Streamwise velocity iso-surfaces (red for positive, blue for negative values) of the real part of the 5 dynamic modes' pairs selected by the sparsity-promoting algorithm, ordered according to their amplitude $|\alpha|$. 


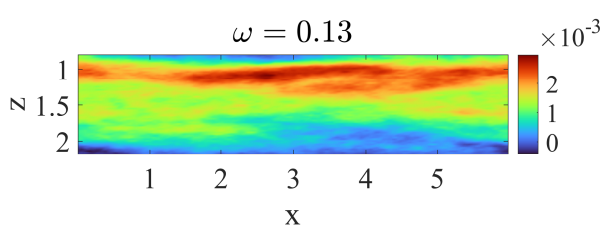

(a) Pair $1, y=-0.41$

$\omega=0.85$

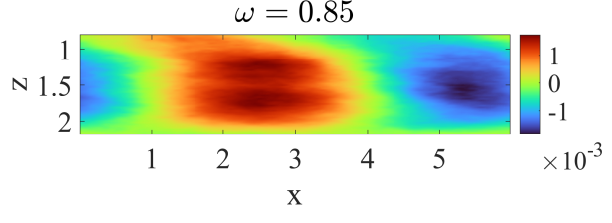

(c) Pair 3, $y=0.41$

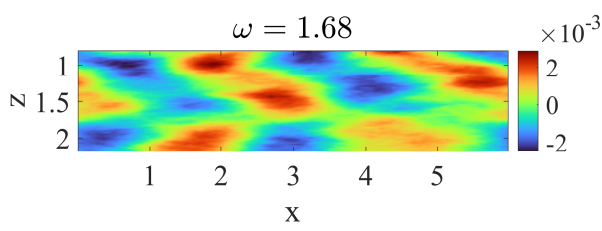

(e) Pair 5, $y=-0.41$

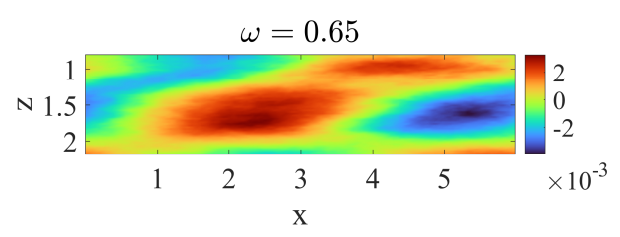

(b) Pair 2, $y=-0.41$

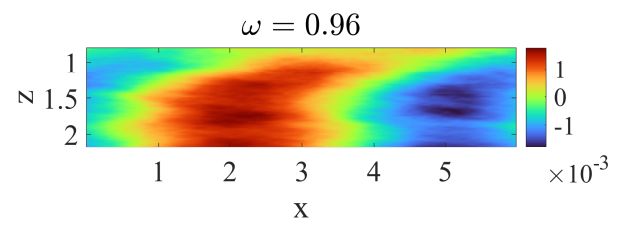

(d) Pair 4, $y=0.41$

Figure 10: Streamwise velocity contours of the selected DMD modes for the precursor simulations in two different $x-z$ planes.

high- and low-speed large-scale streaks of spanwise size $\approx 1$, which are known to constitute one of the two main elements of a self-sustained attached eddy characterizing the large-scale motion in shear flows [35]. Note that, since the DMD is carried out in a subdomain of the precursor computational domain, this DMD mode appears not to be composed of a complete pair of highand low-speed large-scale streaks, but only a portion of the low-speed streak (blue surfaces) is visible in the considered subdomain. Nevertheless, the two-dimensional plots in the $x-z$ and $z-y$ planes, provided in figure 10 (a) and 11 (a), respectively, show the presence of portions of low-speed large-scale streaks on both sizes of the high-speed one, so that the considered DMD mode appears to successfully capture the alternation of low- and high-speed large-scale streaks usually observed in turbulent shear flows. The successive mode, provided in figure 9 (b), is composed of oblique waves localized in the lowest part of the domain. Whereas, the two modes with $\mathfrak{R}(\omega)=0.85,0.96$, provided in figures 9 (c) and (d), respectively, are characterized by streamwise-modulated flow structures with rather large wave length $\left(\lambda_{x} \approx 5\right)$ localized in the upper part of the domain, consistent with an undulation (or secondary instability, see [36]) of the low-frequency elongated streaks, associated with the presence of streamwise vortices. Twodimensional plots of these three modes, provided in figures 10 (b-d) for two $x-z$ planes and figures 11 (b-d) for the $x=2$ plane, show that these DMD modes have a very similar structure (despite being localized in different regions of the domain), and are probably thus linked to the same phenomenon, namely, the secondary instability of large-scale streaks. Finally, among the selected DMD modes, the highest-amplitude one is composed of slightly smaller structures, alternated in the streamwise direction and localized in the lowest part of the domain, as shown in figure 9 (e) and in figures 10 (e) and 11 (e). Notice that the length scales $\left(\lambda_{z} \approx 1-2\right.$, $\lambda_{x} \approx 5-6$ ) of these dynamically relevant flow structures are consistent with those of the large-scale motion observed in turbulent channels and boundary layers [37, 38, 35, 39]. 


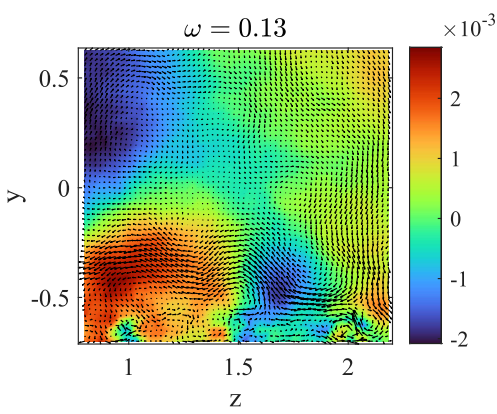

(a) Pair 1

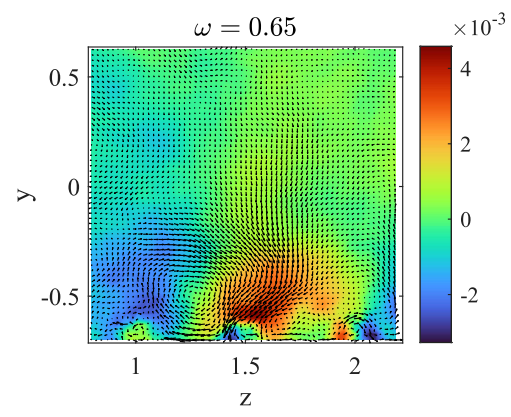

(b) Pair 2

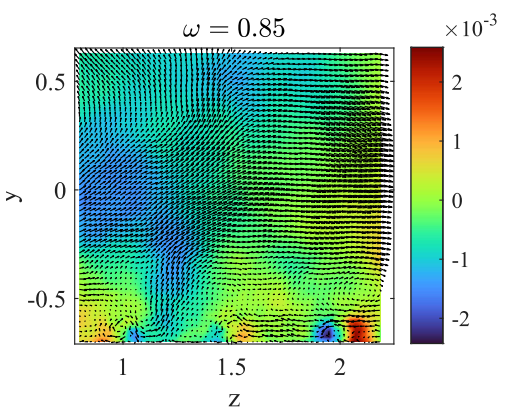

(c) Pair 3

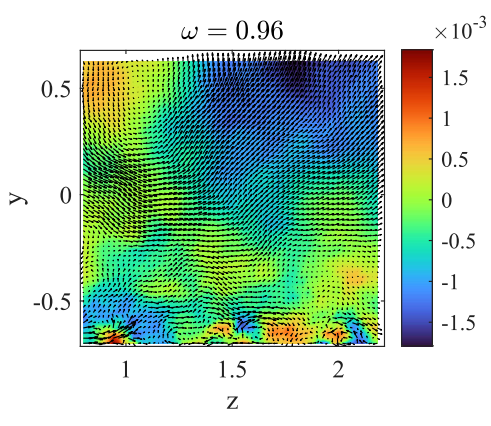

(d) Pair 4

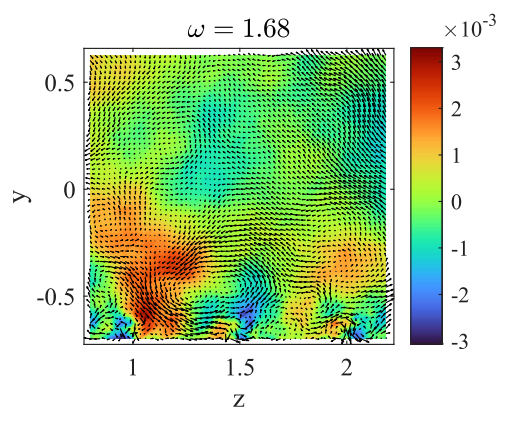

(e) Pair 5

Figure 11: Streamwise velocity contours together with wall-normal and spanwise velocity vectors of the selected DMD modes for the precursor simulations in the $x=2$ plane. 

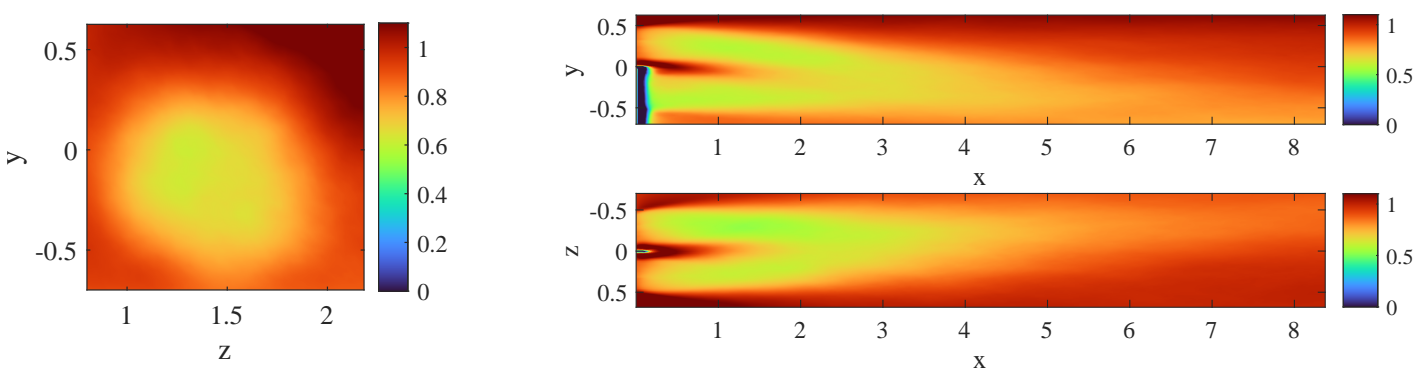

Figure 12: Ensemble average of the LES snapshots of the turbine's wake simulation with inflow turbulence: streamwise velocity in the plane $x=3$ (left), $y=0$ (top right), and $z=0$ (bottom right).

\subsection{Turbine wake with turbulent inflow}

In this section we analyze the DMD of the turbine wake in the presence of the turbulent incoming flow obtained by the precursor simulation. We take snapshots of the velocity field in a reduced three-dimensional subdomain enclosing the wake, with a sampling time corresponding to a $10^{\circ}$ rotation of rotor. As in the previously discussed laminar-inflow case, the extent of the subdomain is $[0-8.4] \times[-0.7-0.63] \times[0.8-2.2]$ in the $x, y$ and $z$ direction, respectively. The entire data-set comprises 3052 snapshots, whose ensemble average (which is subtracted from each snapshot) is shown in Figure 12. One can observe that, due to the turbine rotation, the wake is asymmetric, deviating towards the left in the transverse direction, and towards the bottom in the vertical direction. The presence of a small wake right behind the tower can be highlighted as well. By means of a convergence study, reported in the Appendix, we have found a good convergence of the frequency values for a POD subspace of dimension $r>600$, thus in the following we use $r=601$. In the left panel of Figure 13 the eigenvalues $\mu$ of the linear operator $\boldsymbol{F}$ are shown, along with the unit circle. Note that the growth rate of the modes is not exactly zero (as it should be when the mean flow is subtracted from the snapshots, see [40]) since the DMD is carried out on a projection of the flow fields on a POD subspace. The pairs selected by the sparsity-promoting algorithm for $\gamma=65000$, marked with a black circle in the figure, are seven. Their amplitudes are provided in figure 14. From this figure one can observe that, despite some high-frequency modes (i.e., $\mathfrak{R}(\omega)=28.5-42$ ) can be seen in the standard-DMD amplitude spectrum, these are not selected by the sparsity-promoting algorithm. This indicates that, in the presence of atmospheric turbulence, the high-frequency modes, such as tip vortices, become less relevant for the flow dynamics. The selected SP-DMD modes, ordered by their amplitude, are shown in figure 15. One can notice that all the selected modes, having low frequencies in the range $\mathfrak{R}(\omega) \in[0.37-4.23]$, are associated to largescale spatial structures filling the whole subdomain. Compared with the DMD modes of the laminar-inflow case in figure 5(a, c-e), in the present case the structures have larger wavelengths and are not localized in the far wake but pervade the whole domain. While in the laminarinflow case, the modes displayed a clear geometrical arrangement with respect to the turbine (namely, the tip vortices are localized in the near wake, and the low-frequency ones in the remainder of the domain), in the turbulent-inflow case, no spatial separation among the modes is observed. However, evidence of the presence of the turbine can be seen in some of the selected modes, as shown in figure 16 (c-d-e), since the oscillation clearly appear to be originated at $z=$ $1,1.5,2$, where tip and root vortices are present. On the other hand, many of these modes bear a strong resemblance with those selected by the sparsity-promoting algorithm in the precursor simulation (see figure 9 (b-e)). Concerning their main wavelengths, the DMD modes with 

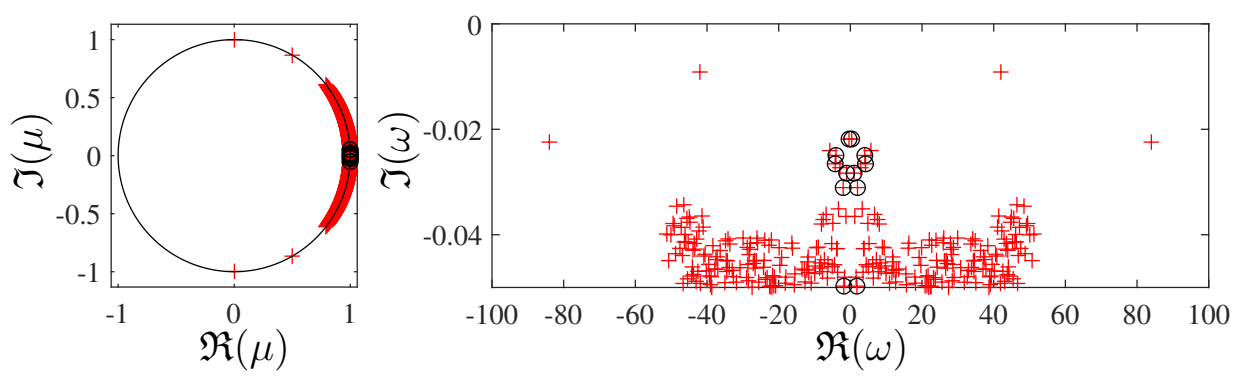

Figure 13: Eigenvalues resulting from the standard DMD algorithm (red crosses) and the sparsity promoting algorithm (black circles) for the case with turbulent inflow. The right panel shows the logarithmic mapping of the eigenvalues, $\omega=-\frac{\log (\mu)}{i \Delta t}$, where $\Delta t$ is the time interval between two consecutive snapshots and $i$ is the imaginary unit.

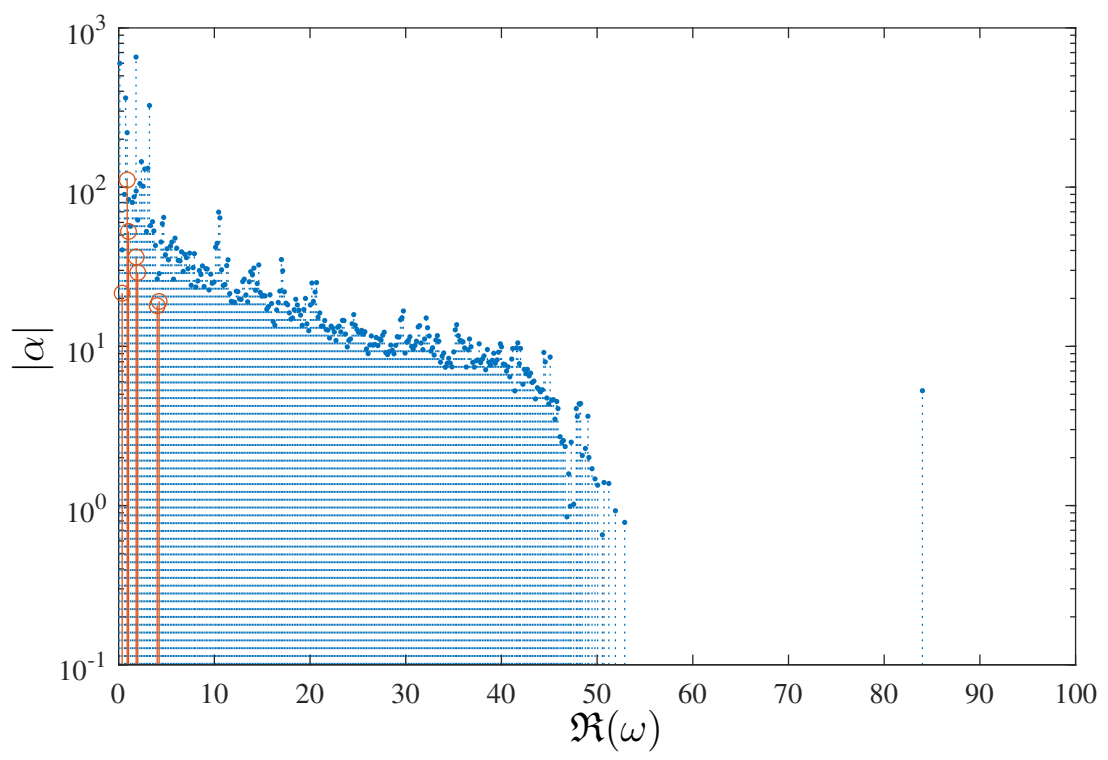

Figure 14: Dependence of the absolute value of the DMD amplitudes $\alpha_{i}$ on the angular frequency $\mathfrak{R}(\omega)$, for the case with turbulent inflow. The results are obtained using the standard (blue stems) and the sparsity-promoting algorithm (orange stems). Due to the symmetry of amplitudes' distribution, only positive frequencies are shown. 


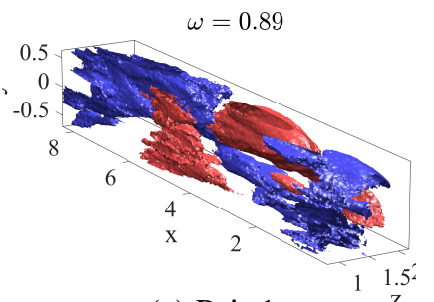

(a) Pair 1

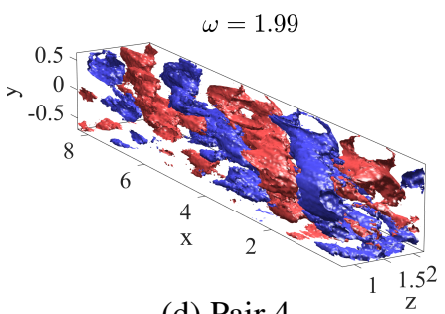

(d) Pair 4

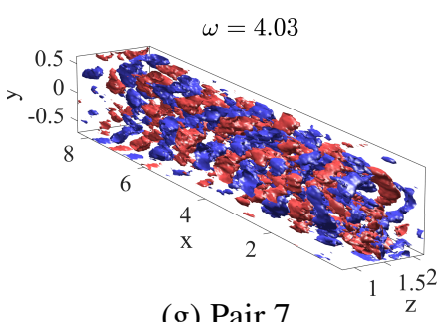

(g) Pair 7

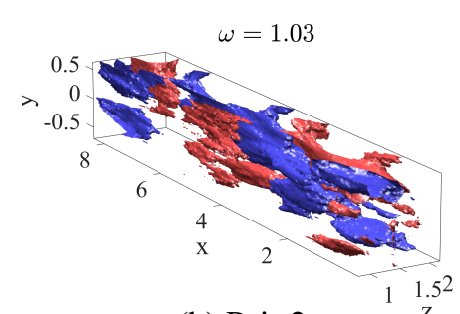

(b) Pair 2

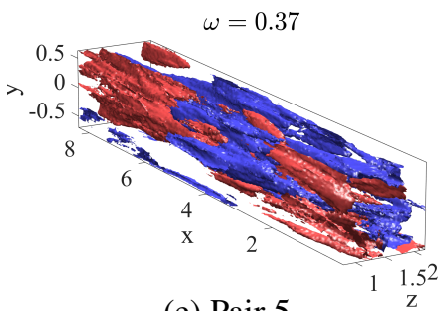

(e) Pair 5

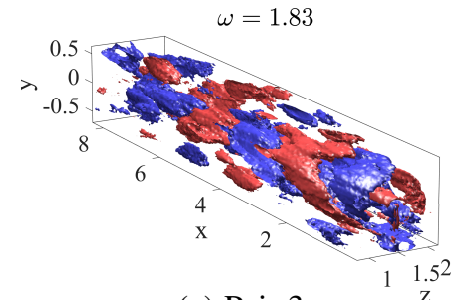

(c) Pair 3

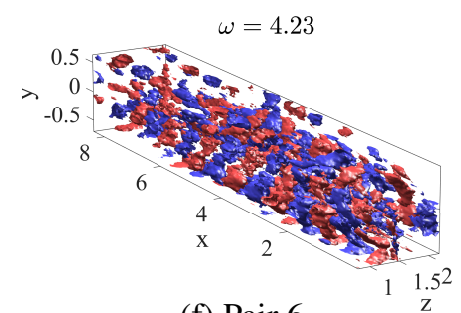

(f) Pair 6

Figure 15: Streamwise velocity iso-surfaces (red for positive, blue for negative values) of the real part of the 8 dynamic modes' pairs selected by the sparsity-promoting algorithm, ordered according to their amplitude $|\alpha|$.

$\mathfrak{R}(\omega)=[0.37-0.89-1.03]$ provided in figure 15 (a-b-e), show streamwise oscillations with $\lambda_{x} \approx 7$ and spanwise size $\approx 1$, similarly to those selected in the precursor simulation (compare also figure 16 (a-b-e) with figure 10 (b-c-d)). Moreover, all the modes characterized by very low frequency $(\Re(\omega) \leq 1)$ have a Strouhal number $S t \leq 0.2$, which lies well within the frequency range typical of the wake meandering [41, 42, 43]. This suggests that wake meandering is due rather to an exogenous mechanism than to an endogenous one, as proposed in recent works [42, 43]. Finer structures are observed for the modes having larger frequencies in the range $\mathfrak{R}(\omega)=[1.83-4.03]$, resembling those found in the laminar-inflow case at similar frequencies (see figure 6 (d-e)), although not presenting a spatial localization in the far wake. The temporal wavenumbers and the streamwise wavelengths of the first five selected SP-DMD modes, compared to those of the laminar-inflow and the precursor simulations, are summarized in table 1 . One can clearly notice that, somehow counter-intuitively, the presence of turbulence at the inflow induces an increase of the main wavelengths from values equal or lower than 2.1, to values larger than $\approx 3.4$.

In order to validate these findings, we have carried out the sparsity-promoting DMD analysis for lower values of $\gamma$, selecting an increasing number of modes for both the laminar and the turbulent inflow cases. The comparison of the resulting DMD spectra, provided in figure 17 (a) for $\approx 50$ modes, clearly shows that, even when many modes are selected, in the turbulentinflow case the high-frequency modes are not selected by the sparsity-promoting DMD algorithm, suggesting that they have lost their dynamical relevance with respect to the laminar inflow case. Moreover, the comparison of the mode amplitudes in figure 17 (b) clearly shows that in the turbulent case the low-frequency modes are associated with much higher amplitudes 


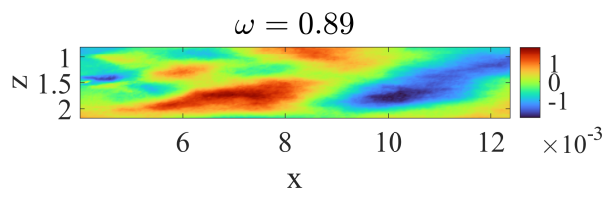

(a) Mode 2 - $y=0.0$

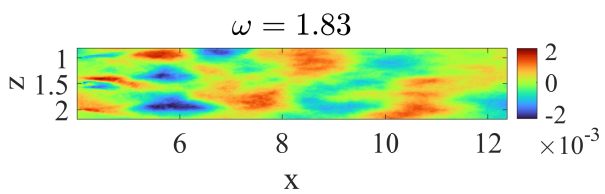

(c) Mode $6-y=0.0$

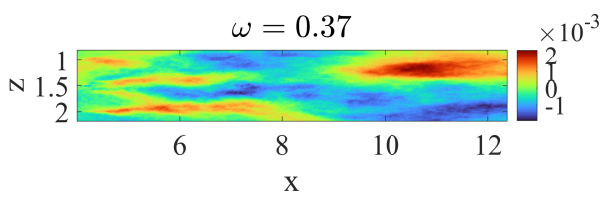

(e) Mode $10-y=0.0$

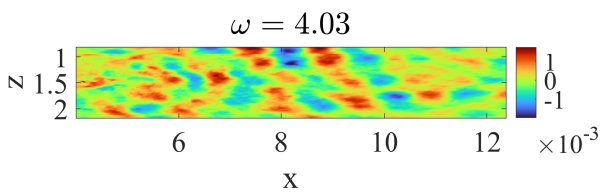

(g) Mode $14-y=0.0$

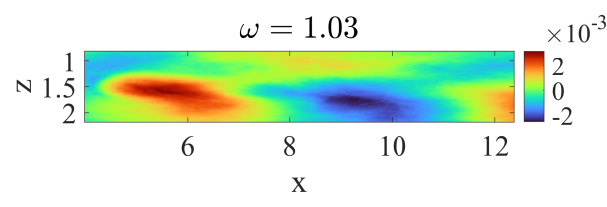

(b) Mode $4-y=0.5$

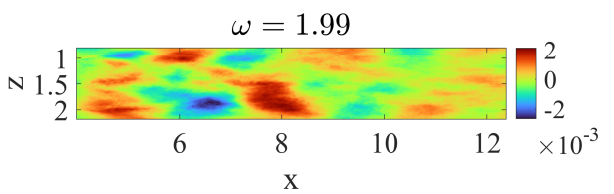

(d) Mode $8-y=0.1$

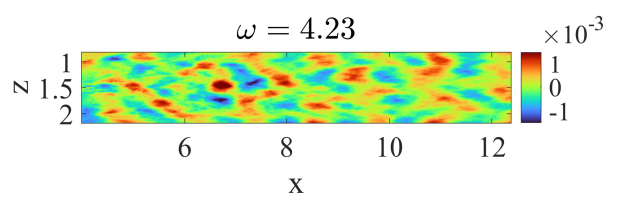

(f) Mode $12-y=0.0$

Figure 16: Streamwise velocity contours of the selected DMD modes for the turbulent inflow simulation in different $x$ - $z$ planes. 
Table 1: Temporal frequencies and main wavelengths of the selected complex conjugate dynamic modes' pairs for the laminar-inflow, the precursor, and the turbulent-inflow simulation.

\begin{tabular}{ccc|cc|cc}
\hline & Laminar inflow & \multicolumn{2}{c}{ Precursor } & \multicolumn{2}{c}{ Turbulent Inflow } \\
\hline & $\mathfrak{R}(\omega)$ & $\lambda_{x}$ & $\mathfrak{R}(\omega)$ & $\lambda_{x}$ & $\mathfrak{R}(\omega)$ & $\lambda_{x}$ \\
\hline Pair 1 & 2.99 & 2.10 & 0.13 & 0 & 0.89 & 7.45 \\
Pair 2 & 42 & 0.17 & 0.65 & 5.32 & 1.03 & 7.45 \\
Pair 3 & 4.98 & 1.41 & 0.85 & 5.32 & 1.83 & 3.39 \\
Pair 4 & 3.2 & 1.68 & 0.96 & 5.32 & 1.99 & 3.59 \\
Pair 5 & 4.81 & 1.41 & 1.68 & 2.62 & 0.37 & 7.45 \\
\hline
\end{tabular}

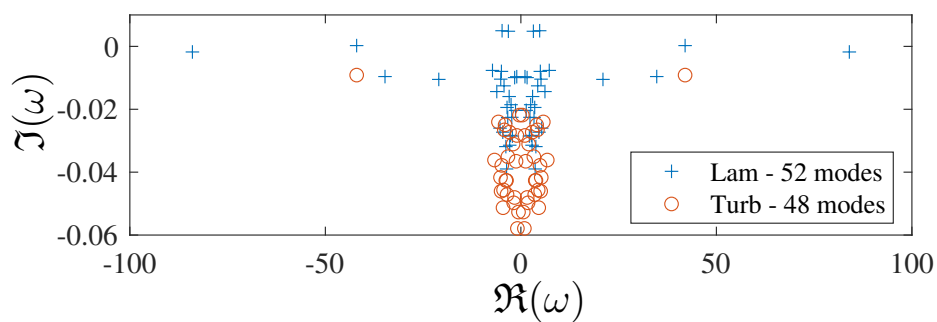

(a)

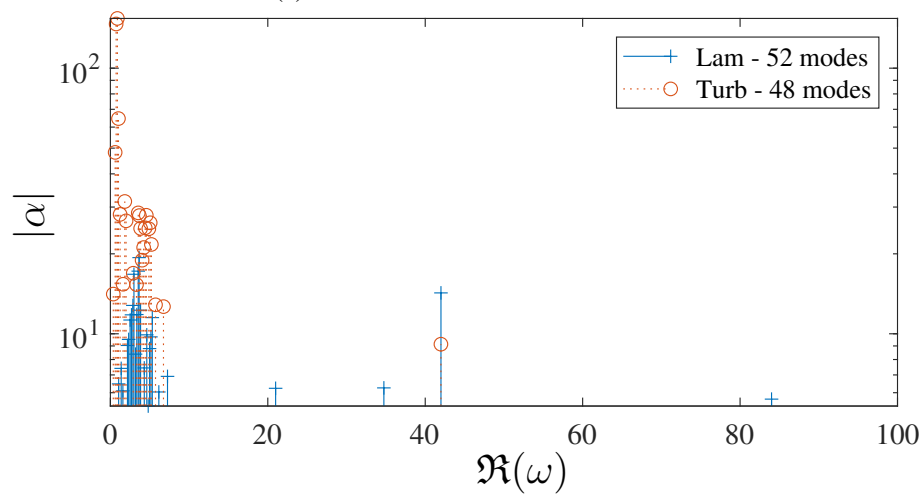

(b)

Figure 17: Comparison between the DMD eigenvalues spectra (a) and the mode amplitudes (b) computed for the cases with laminar and turbulent inflow with a particular $\gamma$ able to select approximately 50 modes in both cases.

than in the laminar one. Similar results have been found for different values of $\gamma$. These results indicate that, in the presence of atmospheric-like turbulence, high-frequency structures, such as tip vortices, loose their dynamical relevance, being superseded by low-frequency modes which have structures and frequencies lying in the range of the wake meandering. Therefore, by the present analysis, we have clearly identified dynamically relevant coherent structures in the turbine wake due to endogenous and exogenous effects. Moreover, the results indicate that large-scale coherent structures with frequencies lying in the range of the wake meandering have an exogenous origin.

\section{Conclusions}

The wake produced by a wind turbine is typically characterized by flow structures originated by endogenous mechanisms, such as the tip- and root-vortex helices or the von Kármán street developing behind the tower. However, when the wind turbine operates in the atmospheric boundary layer, its wake could be significantly affected by flow structures embedded 
in the latter. These flow structures can be detected in the wind turbine wake, even though they are not directly generated by the turbine and are therefore due to exogenous phenomena. The present work investigates the dynamical relevance of coherent structures arising from endogenous and exogenous mechanisms in an NREL-5MW wind turbine's wake under the effect of a turbulent inflow. For the first time, we apply the Sparsity-Promoting Dynamic Mode Decomposition (SP-DMD) to a wind turbine wake; SP-DMD is based on snapshots extracted from large-eddy simulations of the turbine wake, and used for detecting dynamically-relevant flow structures in the presence and in the absence of atmospheric turbulence.

Analysing the selected SP-DMD modes, we demonstrate that inflow turbulence strongly affects the dynamics of the wake, leading to the dynamical prevalence of structures due to exogenous mechanisms and to the detriment of those linked to endogenous ones. When a uniform laminar flow is imposed at the inlet, the most dynamically relevant modes coincide with the high-frequency tip vortices with temporal wavenumber equal to 42 and wavelength $\approx 0.1$, localized in the near wake. Moreover, moderate-scale coherent structures are found, localized in the far wake, having temporal wavenumber $\mathfrak{R}(\omega) \in[3-5]$ and wavelength of order 1. In the presence of inflow turbulence, high-frequency structures, such as the tip vortices, are superseded by large-scale structures having low frequencies in the range $\mathfrak{R}(\omega) \in[0.3-1.6]$ and wavelength $\approx 7$, and by a few moderate-scale modes having higher frequency, $\Re(\omega) \in[3-5]$, and wavelength $\approx 1$, which now fill the whole domain. The DMD analysis of the precursor simulation shows that the low-frequency modes, detected in the turbine wake in the presence of inflow turbulence, roughly correspond to the most dynamically relevant modes for the turbulent flow upstream of the turbine.

These results indicate that structures originating from endogenous mechanisms, such as tip and root vortices, loose their dynamical relevance when turbulence impinges on the turbine, being superseded by large-scale coherent structures with frequencies lying in the range of the wake meandering. SP-DMD has been applied to a wind turbine wake, allowing us to separate endogenous and exogenous effects and to verify that wave meandering is due to exogenous flow fluctuations already present in the atmospheric boundary layer. Thus, accurate ReducedOrder Models (ROM) of utility-scale wind turbines should take into due account the presence of coherent structures embedded in the flow upstream of the turbine. These advanced ROMs will be crucial for: (i) designing modern wind farm with enhanced energy production capacity; (ii) developing real-time active control strategy for "smart" wind farm. Therefore, future work will aim at developing DMD-based reduced-order models in the presence of turbulent inflow.

\section{Appendix A. Convergence with respect to the POD subspace dimension}

The influence of the dimension of the POD subspace on the DMD modes for the laminar inflow case has been investigated in [34], showing a good convergence of the DMD spectrum for a POD subspace dimension $r>300$. Here we show the convergence of the DMD spectrum with respect to $r$ for the precursor simulation (figure 18) and for the turbulent inflow case (figure 19). Figure 18 shows the entire spectrum along with a close-up for the precursor simulation with four values of $r$ ranging from 251 to 401. The close-up in the bottom frame shows that a good convergence of the frequencies is found already for a POD subspace of dimension $r>250$, whereas the growth rates are well approximated for $r>300$. The DMD spectrum for the turbulent inflow case, for values of $r$ ranging from 501 to 701, is shown in figure 19. The results indicate that a good convergence of the frequencies as well as of the growth rate is 


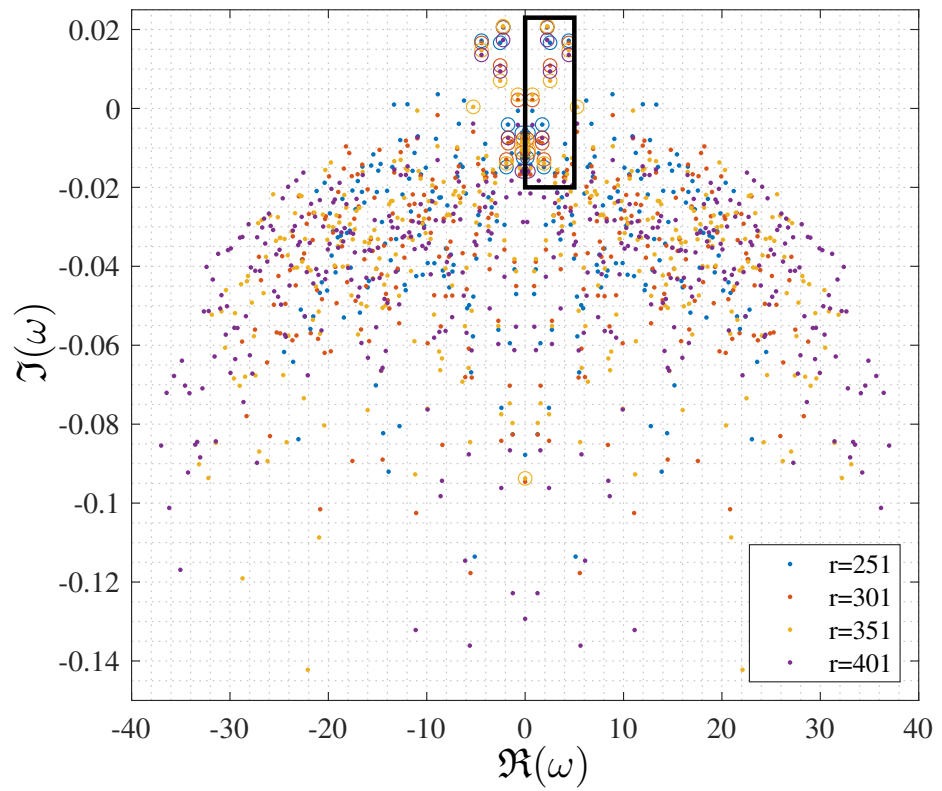

(a)

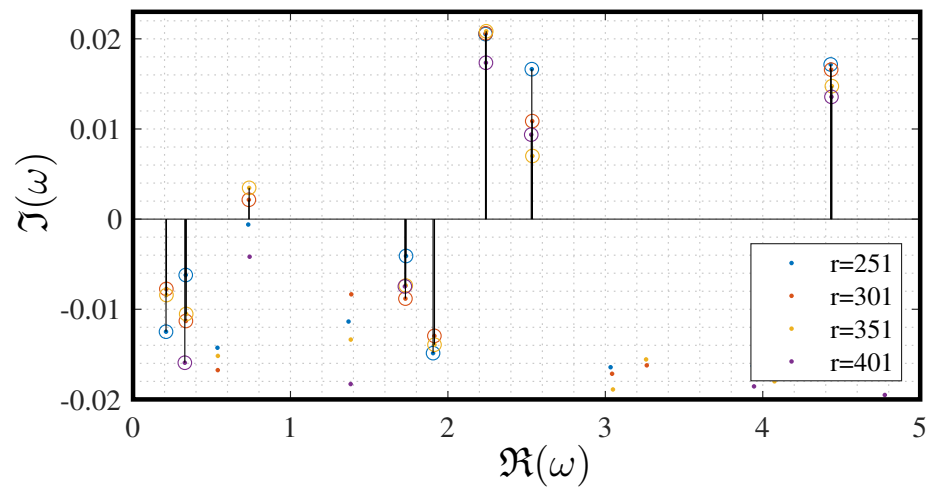

(b)

Figure 18: Convergence of the DMD spectrum with respect to the dimension $r$ of the POD basis for the ABL precursor. 

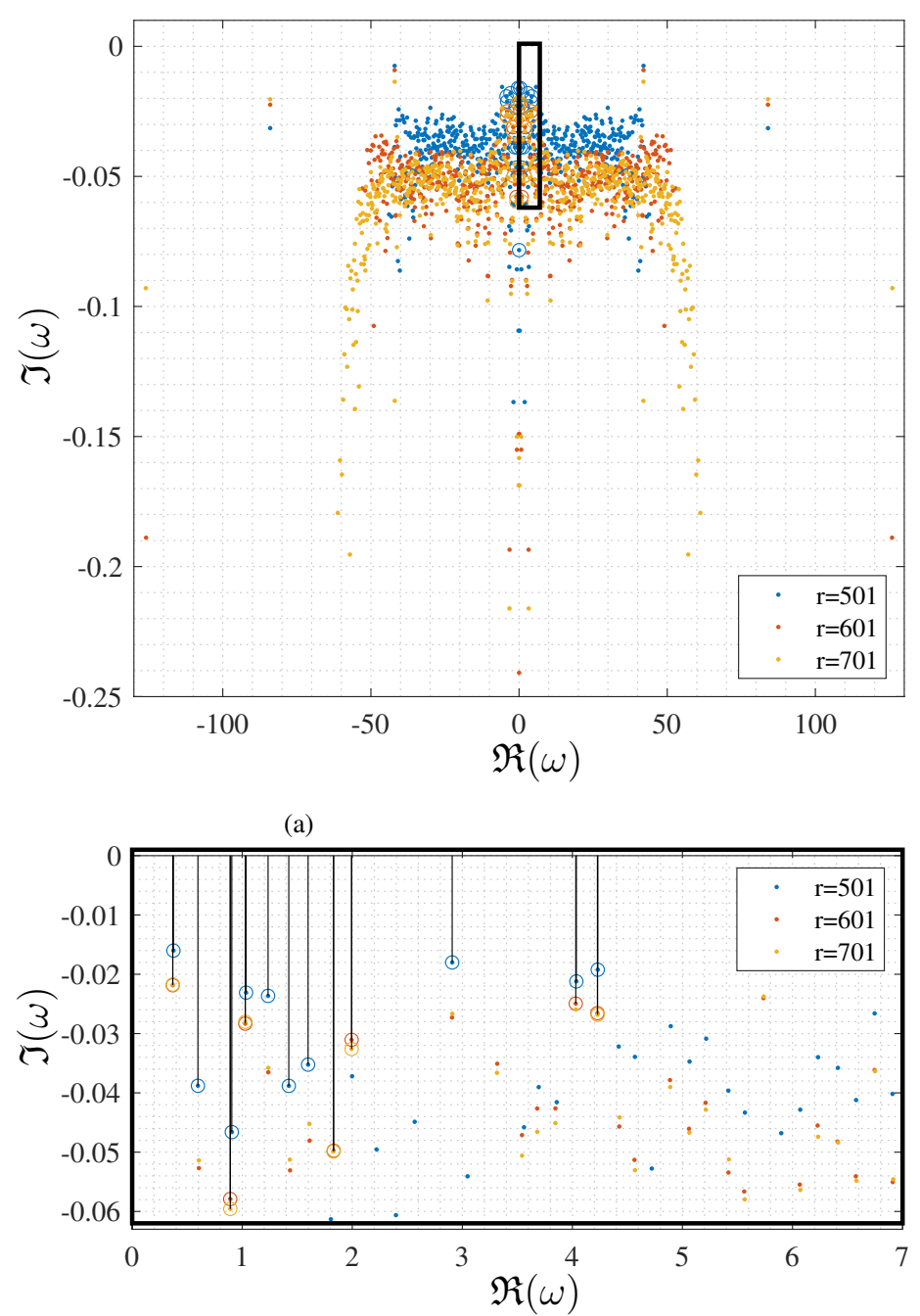

(b)

Figure 19: Convergence of the DMD spectrum with respect to the dimension $r$ of the POD basis for the case with turbulent inlet velocity. 
382 obtained for a POD subspace of dimension $r \geq 601$, which has been chosen for the analysis 383 presented in this work. 


\section{References}

[1] IREA, Future of wind: Deployment, investment, technology, grid integration and socioeconomic aspects (a global energy transformation paper), Technical Report, International Renewable Energy Agency, 2019.

[2] IEA, Renewable 2020, analysis and forecast to 2025. Fuel report., Technical Report, International Energy Agency, 2020.

[3] R. J. Stevens, C. Meneveau, Flow structure and turbulence in wind farms, Annual review of fluid mechanics 49 (2017) 311-339.

[4] F. Porté-Agel, M. Bastankhah, S. Shamsoddin, Wind-turbine and wind-farm flows: A review, Boundary-Layer Meteorology (2019) 1-59.

[5] W. Munters, J. Meyers, An optimal control framework for dynamic induction control of wind farms and their interaction with the atmospheric boundary layer, Phylosophical Transactions A 375 (2017) 20160100.

[6] C. Cossu, Wake redirection at higher axial induction, Wind Energy Science 6 (2021) 377-388.

[7] U. Ciri, M. A. Rotea, S. Leonardi, Effect of the turbine scale on yaw control, Wind Energy 21 (2018) 1395-1405.

[8] E. Kleusberg, S. Bernard, D. S. Henningson, Tip-vortex breakdown of wind turbines subject to shear, Wind Energy 22 (2019) 1789-1799.

[9] N. Troldborg, J. N. Sørensen, R. F. Mikkelsen, Actuator line simulation of wake of wind turbine operating in turbulent inflow, Journal of Physics: Conference Series 75 (2007) 012063.

[10] L. P. Chamorro, F. Porté-Agel, Effects of thermal stability and incoming boundary-layer flow characteristics on wind-turbine wakes: A wind-tunnel study., Boundary-layer meteorology 136 (2010) 515-533.

[11] D. Medici, P. Alfredsson, Measurements on a wind turbine wake: 3D effects and bluff body vortex shedding, Wind Energy: An International Journal for Progress and Applications in Wind Power Conversion Technology 9 (2006) 219-236.

[12] Y.-T. Wu, F. Porté-Agel, Atmospheric turbulence effects on wind-turbine wakes: An LES study, energies 5 (2012) 5340-5362.

[13] J. Jonkman, S. Butterfield, W. Musial, G. Scott, Definition of a 5-MW refrence wind turbine for offshore system development, Technical Report 38060, NREL-TP-500-38060, 2009.

[14] P. J. Schmid, Dynamic mode decomposition of numerical and experimental data, Journal of Fluid Mechanics 656 (2010) 5-28. 
[15] C. W. Rowley, I. Mezić, S. Bagheri, P. Schlatter, D. S. Henningson, Spectral analysis of nonlinear flows, Journal of Fluid Mechanics 641 (2009) 115-127.

[16] G. V. Iungo, C. Santoni-Ortiz, M. Abkar, F. Porté-Agel, M. A. Rotea, S. Leonardi, Datadriven reduced order model for prediction of wind turbine wakes, Journal of Physics: Conference Series 625 (2015) 012009.

[17] M. Debnath, C. Santoni, S. Leonardi, G. V. Iungo, Towards reduced order modelling for predicting the dynamics of coherent vorticity structures within wind turbine wakes, Philosophical Transactions of the Royal Society A: Mathematical, Physical and Engineering Sciences 375 (2017).

[18] S. Le Clainche, L. S. Lorente, J. M. Vega, Wind predictions upstream wind turbines from a lidar database, Energies 11 (2018) 543.

[19] J. N. Kutz, S. L. Brunton, B. W. Brunton, J. L. Proctor, Dynamic mode decomposition: data-driven modeling of complex systems, SIAM, 2016.

[20] S. Sarmast, R. Dadfar, R. F. Mikkelsen, P. Schlatter, S. Ivanell, J. N. Sørensen, D. S. Henningson, Mutual inductance instability of the tip vortices behind a wind turbine, Journal of Fluid Mechanics 755 (2014) 705-731.

[21] P. Premaratne, H. Hu, Analysis of turbine wake characteristics using dynamic mode decomposition, in: 35th AIAA Applied Aerodynamics Conference, AIAA, 2017, pp. 2017-4214.

[22] V. G. Kleine, E. Kleusberg, A. Hanifi, D. S. Henningson, Tip-vortex instabilities of two in-line wind turbines, in: Journal of Physics: Conference Series, volume 1256, IOP Publishing, 2019, p. 012015.

[23] C. Sun, T. Tian, X. Zhu, O. Hua, Z. Du, Investigation of the near wake of a horizontal-axis wind turbine model by dynamic mode decomposition, Energy 227 (2021) 120418.

[24] M. R. Jovanović, P. J. Schmid, J. W. Nichols, Sparsity-promoting dynamic mode decomposition, Physics of Fluids 26 (2014) 024103.

[25] C. Santoni, K. Carrasquillo, I. Arenas-Navarro, S. Leonardi, Effect of tower and nacelle on the flow past a wind turbine, Wind Energy 20 (2017) 1927-1939.

[26] G. De Cillis, S. Cherubini, O. Semeraro, S. Leonardi, P. De Palma, POD-based analysis of a wind turbine wake under the influence of tower and nacelle, Wind Energy 24 (2021) 609-633.

[27] W.-C. Cheng, F. Porté-Agel, Adjustment of turbulent boundary-layer flow to idealized urban surfaces: A large-eddy simulation study, Boundary-Layer Meteorology 155 (2015) 249-270.

[28] P. Orlandi, Fluid flow phenomena: a numerical toolkit, volume 55, Springer Science \& Business Media, 2012. 
[29] J. N. Sørensen, W. Z. Shen, Computation of wind turbine wakes using combined navierstokes/actuator-line methodology, in: 1999 European Wind Energy Conference and Exhibition, 1999, pp. 156-159.

[30] M. D. de Tullio, P. De Palma, G. Iaccarino, G. Pascazio, M. Napolitano, An immersed boundary method for compressible flows using local grid refinement, Journal of Computational Physics 225 (2007) 2098-2117.

[31] P. Orlandi, S. Leonardi, DNS of turbulent channel flows with two-and three-dimensional roughness, Journal of Turbulence (2006) N73.

[32] C. Santoni, E. J. García-Cartagena, U. Ciri, L. Zhan, G. V. Iungo, S. Leonardi, One-way mesoscale-microscale coupling for simulating a wind farm in north texas: Assessment against SCADA and LiDAR data, Wind Energy 23 (2021) 691-710.

[33] L. Sirovich, Turbulence and the dynamics of coherent structures. Parts i-iii., Quarterly of applied mathematics 45 (1987) 561-590.

[34] G. De Cillis, S. Cherubini, O. Semeraro, S. Leonardi, P. De Palma, DMD of the wake of a NREL-5MW wind turbine, SDEWES Conference submitted to (2021).

[35] Y. Hwang, Statistical structure of self-sustaining attached eddies in turbulent channel flow, Journal of Fluid Mechanics 767 (2015) 254-289.

[36] M. de Giovanetti, H. J. Sung, Y. Hwang, Streak instability in turbulent channel flow: the seeding mechanism of large-scale motions, Journal of Fluid Mechanics 832 (2017) $483-513$.

[37] L. S. G. Kovasznay, V. Kibens, R. F. Blackwelder, Large-scale motion in the intermittent region of a turbulent boundary layer, Journal of Fluid Mechanics 41 (1970) 283-325.

[38] J. Lee, J. Lee, J. Choi, H. Sung, Spatial organization of large- and very-large-scale motions in a turbulent channel flow, Journal of Fluid Mechanics 749 (2014) 818-840.

[39] M. Farano, S. Cherubini, P. De Palma, J.-C. Robinet, Nonlinear optimal large-scale structures in turbulent channel flow, European Journal of Mechanics - B/Fluids 72 (2018) 74-86.

[40] K. K. Chen, J. H. Tu, C. W. Rowley, Variants of dynamic mode decomposition: Boundary condition, Koopman, and Fourier analyses., Journal of Nonlinear Science 22 (2012) 887-915.

[41] D. Medici, Experimental studies of wind turbine wakes: power optimisation and meandering, Ph.D. thesis, KTH, 2005.

[42] X. Mao, J. Sørensen, Far-wake meandering induced by atmospheric eddies in flow past a wind turbine, Journal of Fluid Mechanics 846 (2018) 190-209.

[43] V. Gupta, M. Wan, Low-order modelling of wake meandering behind turbines, Journal of Fluid Mechanics 877 (2019) 534-560. 